\begin{tabular}{|c|c|}
\hline Title & Smooth epitaxial copper film on sapphire surface suitable for high quality graphene growth \\
\hline Author(s) & Ma, Tao; A riga, Hiroko; Takakusagi, Satoru; A sakura, Kiyotaka \\
\hline Citation & $\begin{array}{l}\text { Thin solid films, 646, 12-16 } \\
\text { https://doi.org/10.1016/.tsf.2017.11.009 }\end{array}$ \\
\hline Issue Date & 2018-01-31 \\
\hline Doc URL & http:/hdl.handle.net/2115/76637 \\
\hline Rights & 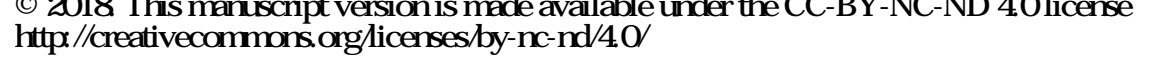 \\
\hline Rights(URL) & http://creativecommons.org/icenses/by-nc-nd/4.0/ \\
\hline Type & article (author version) \\
\hline File Information & elsivier1025T A 01.pdf \\
\hline
\end{tabular}

Instructions for use 


\title{
Smooth Epitaxial Copper Film on Sapphire Surface Suitable for High Quality Graphene Growth
}

\author{
Tao Ma, Hiroko Ariga, Satoru Takakusagi, Kiyotaka Asakura* \\ Institute for Catalysis, Hokkaido University \\ N21 W10, Sapporo, Hokkaido 001-0021, Japan
}

\begin{abstract}
Graphene, a two-dimensional material, can be grown on a metal substrate using chemical vapor deposition - this growth process is notably influenced by the crystal orientation and the roughness of the substrate surface. We prepared epitaxial $\mathrm{Cu}(111)$ films on sapphire substrates using thermal evaporation at various substrate temperatures and studied their crystal orientation and roughness. The well crystallized $\mathrm{Cu}(111)$ film with a smooth surface was obtained when the substrate was maintained at $473 \mathrm{~K}$ during the deposition. High quality graphene with few intrinsic defects was grown on this $\mathrm{Cu}$ film.
\end{abstract}

Keywords: Epitaxial growth; $\mathrm{Cu}(111)$ films; crystal orientation; roughness; chemical vapor deposition; defects.

\section{Introduction}

Graphene, a two-dimensional carbon material composed by a single graphite layer, shows extraordinary electronic, thermal and mechanical properties [1-3]. In our group, we are developing an environment-controlled cell with a graphene 5 window for in-situ electron microscopy and spectroscopy. The graphene window with clean and defectless area in the sub-millimeter scale is desired to ensure optimal signal collection. In our previous work, we have conquered the first step to transfer chemical vapor deposition (CVD) graphene without bringing considerable contamination to the film [4].

10 Graphene prepared by CVD with comercial copper foils is usually polycrystalline, with the domain size within the micrometer scale [5-7]. It was reported that the domain size of the CVD graphene could be notably influenced by the domain size and the roughness of employed metal substrates [8, 9]. Surface defects such as grain boundaries in the commercial polycrystalline copper can 15 be the nucleation sites for multiple graphene domains $[9,10]$, leading to polycrystalline graphene with small domains. Single-crystalline metal substrates, in

\footnotetext{
* Corresponding author

Email address: askr@cat.hokudai.ac.jp (Kiyotaka Asakura)
} 
which the grain boundaries are absent, can significantly reduce the nucleation sites and hence produce single-crystalline graphene with relatively large area [11-13]. However, the single-crystalline metals are expensive and impractical with a chemical solution. On the other hand, large-scale graphene with reduced defects could be prepared on metal thin films with smooth surface [14, 15]. Procházka et al. demonstrated that the graphene layer grown on an ultrasmooth copper film, which was deposited on a $\mathrm{SiO}_{2} / \mathrm{Si}$ substrate, had a very

25 low amount of defects, evident by the absence of D-band in the Raman spectra [14].

Recently, an easy way to prepare smoothly and uniformly oriented copper films on single-crystalline sapphire has been reported [16-19]. Because the crystal orientations of copper substrates can remarkably alter the growth dynam30 ics of graphene [18], copper films with a uniform crystal orientation eliminate the diverse growth behavior of the graphene domains, providing chances to grow large-scale single-crystalline graphene. It has already been confirmed that graphene prepared on the $\mathrm{Cu}(111)$ /sapphire substrate was almost free of defects [16], and had a single domain with an area over $1 \mathrm{~mm}^{2}$ [17]. However, these

35 works are focused on either the growth or the characterization of the graphene films, whereas the methodology of the preparation of the epitaxial substrates were hardly studied.

In order to optimize the preparation of epitaxial copper films on sapphire, we investigated the crystal orientation and the roughness of copper films pre40 pared at various substrate temperatures. The optimized sample was selected for graphene growth via CVD, with the results compared with that grown on a commercial copper foil. Our results showed that the $\mathrm{Cu} /$ sapphire substrate has a potential to prepare high quality graphene.

\section{Experimental Procedures}

45

Copper films were deposited on the $c$-plane of sapphire, $\alpha-\mathrm{Al}_{2} \mathrm{O}_{3}(0001)(10$ $\mathrm{mm} \times 10 \mathrm{~mm} \times 0.5 \mathrm{~mm}$, Shinkosha Co., Ltd), using thermal evaporation. Copper beads (2-8 mm, 99.99\%, Sigma-Aldrich) were put on a tantalum boat that was resistively heated inside the evaporator. During the evaporation, the sapphire substrate was heated at varied temperatures from $473 \mathrm{~K}$ to $623 \mathrm{~K}$ using a platinum wire that was mounted close to the substrate. The substrate temperature was monitored by a thermocouple that was attached to the back side of the sapphire substrate. The evaporation rate, monitored by a quartz crystal unit, was kept to $0.2 \mathrm{~nm} / \mathrm{min}$ until the thickness of the deposited film reached to $\sim 750 \mathrm{~nm}$. We also evaporated the copper film without heating the 55 substrate externally, which was denoted as the room-temperature (RT) sample; but note that the substrate was warmed radiatively by the evaporating source and its temperature was gradually raised to $\sim 473 \mathrm{~K}$ when the deposition finished. The crystallography of the copper films was studied by X-ray diffraction (XRD; Bruker D8 Discover) with $\mathrm{Cu} \mathrm{K}_{\alpha}$ radiation: $\theta-2 \theta$ scans were recorded in 60 a range of $2 \theta=30^{\circ}-80^{\circ}$, which covers the main peaks of a face-centered cubic 

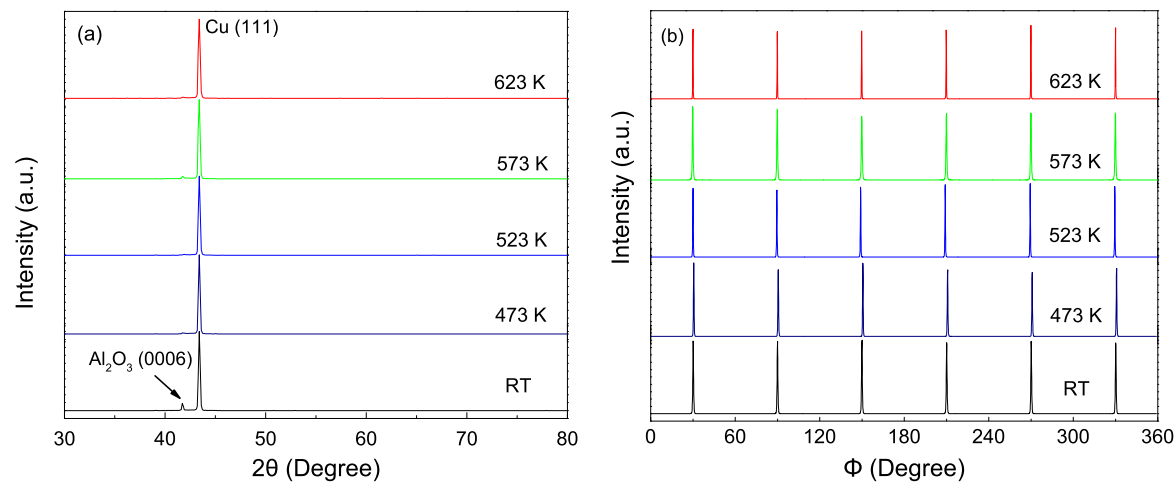

Figure 1: XRD profiles of the $\mathrm{Cu}(111)$ films on $\mathrm{Al}_{2} \mathrm{O}_{3}(001)$ deposited under varied substrate temperatures. The $\theta-2 \theta$ scans (a) show only (111) reflections from the copper films; The $\phi$-scans (b) show $\mathrm{Cu}\{111\}$ diffractions appeared with an interval of $60^{\circ}$.

structure. The [111]-pole $\phi$-scans were performed with the optical configuration of $2 \theta=43.4^{\circ}, \chi=70.5^{\circ}$, and rotating the sample normal to the surface by $\phi=0^{\circ}-360^{\circ}$. The texture of the films normal to the surface were recorded by electron backscattering diffraction (EBSD; JEOL JIB-4600F/HKD), with a

${ }_{65}$ mapping step of $0.2 \mu \mathrm{m}$ in a $10 \times 40 \mu \mathrm{m}^{2}$ area. The morphology and the roughness of the films were examined using atomic force microscopy (AFM, Asylum Technology).

Graphene was grown on the copper films using ambient pressure CVD. The prepared $\mathrm{Cu}(111) / \mathrm{Al}_{2} \mathrm{O}_{3}(0001)$ was inserted into the CVD reactor, which was then heated to $1000{ }^{\circ} \mathrm{C}$ within 40 min in Ar-blended $\mathrm{H}_{2}$ (3.95 vol.\%) flowing at a rate of $50 \mathrm{sccm}$, and maintained for $20 \mathrm{~min}$. Then Ar-blended $\mathrm{CH}_{4}$ (0.987 vol.\%) was introduced at a rate of $10 \mathrm{sccm}$ together with the $\mathrm{H}_{2} /$ Ar flowing at $50 \mathrm{sccm}$ for a period of $60 \mathrm{~min}$ to grow graphene. The sample was then gradually cooled down to room temperature inside the reactor, with the $\mathrm{CH}_{4} / \mathrm{Ar}$ and $\mathrm{H}_{2} / \mathrm{Ar}$ 75 gases still flowing. The total pressure inside the reactor was kept at $95 \mathrm{kPa}$ in the entire process to reduce the dewetting of the copper film. The prepared graphene was compared with the typical CVD graphene grown on a commercial copper foil with a slightly modified recipe, reported in our previous work [4]. Raman spectroscopy with a $532 \mathrm{~nm}$ laser (Renishaw inVia Reflex), as well as

so scanning electron microscopy (SEM; JEOL JSM-7400F), was used to estimate the quality of the graphene layer.

\section{Results and Discussion}

Figure 1a shows the $\theta-2 \theta$ XRD profiles of the copper films deposited on the sapphire at different substrate temperatures. Only the reflections from $\mathrm{Cu}(111)$ and $\mathrm{Al}_{2} \mathrm{O}_{3}(0006)$ were detected in all the samples, indicating that the copper films were well grown along (111), with the orientation preference of 
$\mathrm{Cu}(111) \| \mathrm{Al}_{2} \mathrm{O}_{3}(0001)$. To further confirm this epitaxial growth, we performed the [111]-pole $\phi$-scans by fixing the X-ray optics at the Bragg position of $\mathrm{Cu}(111)$ $\left(2 \theta=43.5^{\circ}\right)$, tilting the sample by $70.5^{\circ}$ away from the horizontal plane, and rotating the samples along the [111]-pole that is normal to the surface. The resulted spectra show diffractions from the (111), (1111), and (111) planes, which are $70.5^{\circ}$ away from the (111) surface. As presented in figure $1 \mathrm{~b}$, distinct peaks appear periodically with an interval of $60^{\circ}$, indicating that the copper films were epitaxially grown on the $c$-plane sapphire. No obvious difference in the 95 spectra was seen when we altered the substrate temperatures, revealing that the epitaxial $\mathrm{Cu}(111)$ film could be prepared with the sapphire substrate temperatures ranging from RT to $623 \mathrm{~K}$. Note that the $\phi$-scan diffraction patterns show a sixfold symmetry in the copper films, whereas the single-crystalline copper has a threefold symmetry along [111] pole. Therefore, it is likely that the deposited films are composed of a two-domain structure with the crystallites rotated azimuthally by $60^{\circ}[20]$. It is worth mentioning that the RT sample here clearly showed the epitaxy feature, in contrast to the results of Bialas et al., who reported that the epitaxial copper film was not obtained when the substrate was maintained at $298 \mathrm{~K}$ [20]. The difference may arise because the 105 substrate, though not intentionally heated, was warmed by the evaporation source during the deposition. Thus, we speculate that the epitaxial film in the RT sample started to form when the substrate temperature surpassed a certain threshold between RT to $473 \mathrm{~K}$. It is possible that there is a small amount of polycrystalline or amorphous copper formed in the initial stage when the substrate temperature was below the threshold (e.g., at RT).

To confirm this two-domain structure in the copper films, we carried out EBSD measurements on these samples. As shown in Figure 2, the scanned area in all the samples was indexed by the blue color that corresponded to $\mathrm{Cu}(111)$ orientation. The black lines indicate the boundaries between the domains with

115 (111) surface but twisted by $60^{\circ}$ azimuthally. Note that there are a small amount of misoriented domains ( $\sim 1 \mu \mathrm{m}$ in size) in the RT sample, agreeing with our speculation that polycrystalline copper formed in the initial stage of deposition. In addition, the size of the two-domain grains gradually increased with the elevation of the substrate temperature. This can be explained by the Volmer-

${ }_{120}$ Weber [21] model where a continuous film is created by the formation of small islands at the initial stage, followed by an island-coalescence process [20, 22]. At higher temperatures, the growth of the copper islands is facilitated, leading to a larger grain size compared to lower temperatures. However, the rapid threedimensional growth of the copper islands at high temperature can also lead to 125 the formation of other facets like (100) and (110), resulting in the mis-oriented domains in the copper film, as shown in panel (e).

Figure 3 shows the AFM images of the copper films prepared under various substrate temperatures. The numbers in the figures are the root mean square (RMS) as an indication of the surface roughness. The deposited films show 130 the similar surface morphology with small grains along (111). Those grains coarsen as the substrate temperature increases, in agreement with the EBSD observation. Accordingly, the roughness of the copper films also increases with 


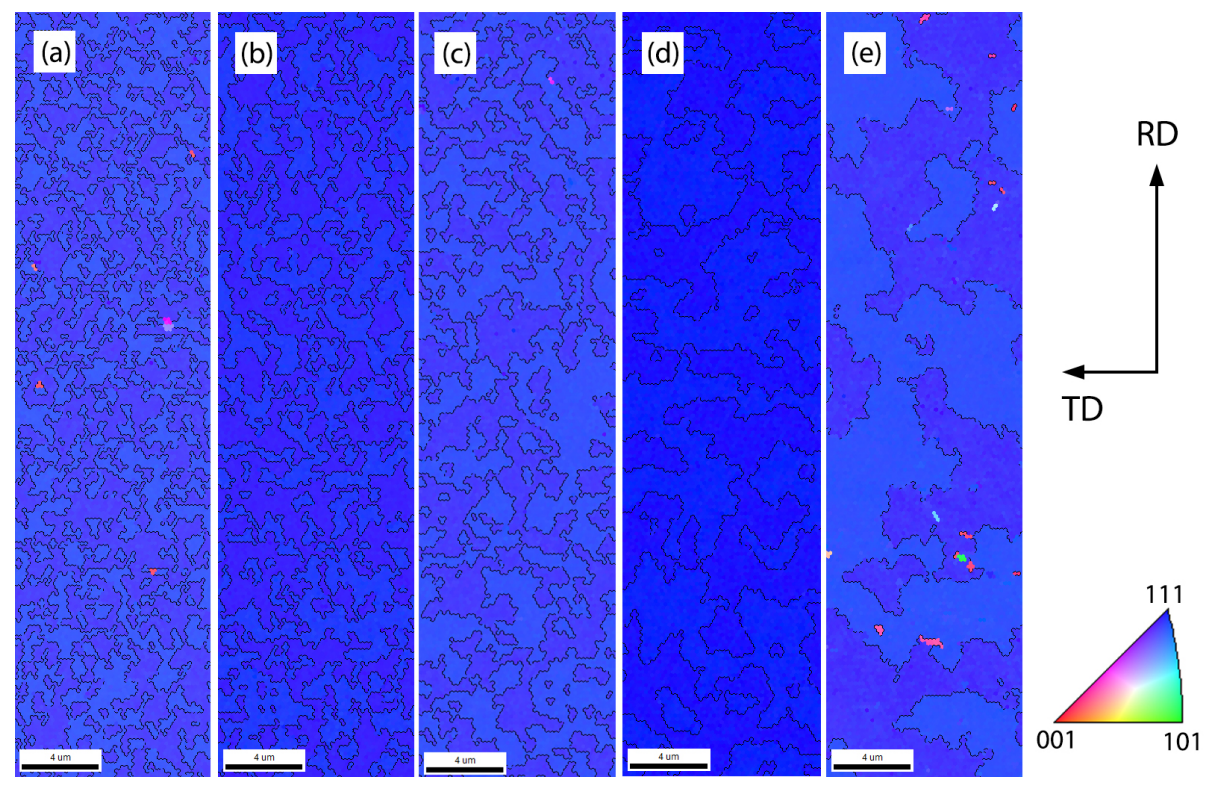

Figure 2: EBSD images of the $\mathrm{Cu}(111)$ films evaporated epitaxially on $\mathrm{Al}_{2} \mathrm{O}_{3}(0001)$ when the substrate was initially maintained at RT (a), $473 \mathrm{~K}(\mathrm{~b}), 523 \mathrm{~K}$ (c), $573 \mathrm{~K}$ (d), and $623 \mathrm{~K}$ (e). The scale bar is $4 \mu \mathrm{m}$.

the elevation of the substrate temperature, e.g., RMS $=7.2 \mathrm{~nm}$ at $473 \mathrm{~K}$, whereas RMS $=37.3 \mathrm{~nm}$ at $623 \mathrm{~K}$, as a result of the grain coarsening. For 135 comparison, a commercial copper foil which is commonly used for CVD graphene growth was also scanned by AFM, as shown in Figure 3f. The RMS of the foil was estimated to be $41.3 \mathrm{~nm}$. By growing $\mathrm{Cu}$ films epitaxially on sapphire, we were able to reduce the roughness to $\sim 1 / 6$ of that of the commercial copper foil.

The above results showed that the $\mathrm{Cu}(111)$ films were grown epitaxially on $\mathrm{Al}_{2} \mathrm{O}_{3}(0001)$, with the roughness and crystal orientation depending on the temperature of $\mathrm{Al}_{2} \mathrm{O}_{3}$ substrate. At very low temperature (RT sample), the deposited $\mathrm{Cu}$ atoms had very low mobility. Thus, the growth of the $\mathrm{Cu}$ film was dominated by the nucleation of small $\mathrm{Cu}$ islands, resulting in the morphology with small domains. Also, it is likely that the film has variation in the thickness, again, due to the low mobility of the $\mathrm{Cu}$ atoms. This can be inferred from the existence of $\mathrm{Al}_{2} \mathrm{O}_{3}(0006)$ peak in the XRD pattern of the RT sample, as the diffracted X-ray reached to the detector from some thin area of the copper film. The combination of these two factors led to the rough surface of the copper film. When the substrate temperature was raised to $473 \mathrm{~K}$, the migration of ${ }_{150}$ the $\mathrm{Cu}$ atoms was enhanced. The copper nuclei then could grow epitaxially on the $\mathrm{Al}_{2} \mathrm{O}_{3}$ substrate and form a flat surface to reduce the surface tension. Therefore, the surface roughness was ameliorated. At higher temperature, the mobility of the $\mathrm{Cu}$ atoms could be very high. As a result, the film growth was 

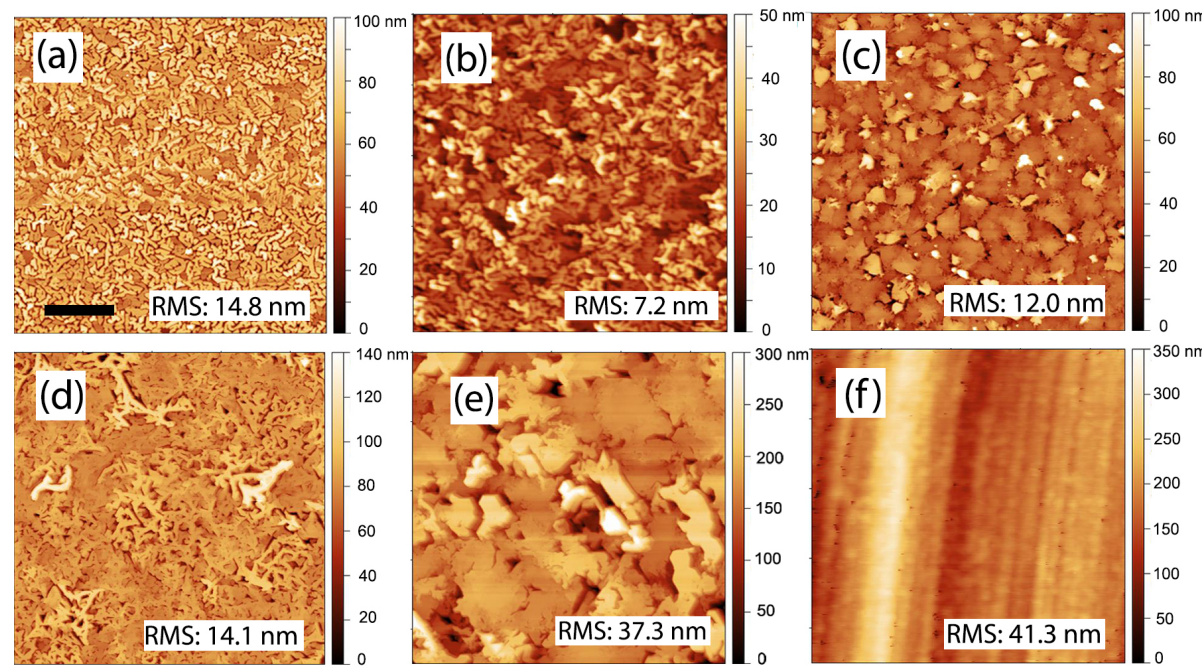

Figure 3: AFM images of the $\mathrm{Cu}(111)$ films deposited epitaxially on $\mathrm{Al}_{2} \mathrm{O}_{3}(0001)$ when the substrate was initially maintained at RT (a), $473 \mathrm{~K}(\mathrm{~b}), 523 \mathrm{~K}(\mathrm{c}), 573 \mathrm{~K}$ (d), and $623 \mathrm{~K}(\mathrm{e})$. A commercial copper foil (f) was also scanned for comparison. The scale bar is $2 \mu \mathrm{m}$. The roughness of the samples was estimated by the RMS values shown in the figures.

dominated by the growth of the copper islands, which could take place in a

three-dimensional way due to the fast migration of the $\mathrm{Cu}$ atoms. In this case, the epitaxial relationship between copper and the substrate was not always maintained. Other $\mathrm{Cu}$ facets could be formed during the three-dimensional growth and further coalescence of the copper islands, leading to the increase of the surface roughness.

We attempted to grow graphene on the $\mathrm{Cu}(111) / \mathrm{Al}_{2} \mathrm{O}_{3}(0001)$ film prepared at the substrate temperature of $473 \mathrm{~K}$, which possesses the smoothest surface among all the epitaxial films studied in this work. Raman spectroscopy was used to evaluate the quality of the graphene film, under the consideration that the $\mathrm{D}$ band at $\sim 1350 \mathrm{~cm}^{-1}$ is a direct indicator of defects in graphene [16, 23]. Figure

1654 shows three spectra randomly obtained from different spots in graphene on the $\mathrm{Cu}(111) / \mathrm{Al}_{2} \mathrm{O}_{3}(0001)$. Except for the distinct $\mathrm{G}$ band at $\sim 1580 \mathrm{~cm}^{-1}$ and $2 \mathrm{D}$ band at $\sim 2680 \mathrm{~cm}^{-1}$, no obvious peaks could be found in the $\mathrm{D}$ band region, revealing the low density of intrinsic defects (below the detection limit) in the graphene film. Note that the noise in the background was from the copper substrate as the spectra were collected without further transfer of graphene to other substrates, whereas graphene grown on copper foils was transferred to $\mathrm{SiO}_{2}$ to reduce the noise in the Raman spectra. Figure 5a shows the morphology of the epitaxial copper film after graphene growth. It can be seen that the contrast was uniform in the most part, indicating that the graphene layer grown on the film had uniform thickness in a large area. Only some small area near the domain boundaries showed dark contrast, corresponding to thicker regions 


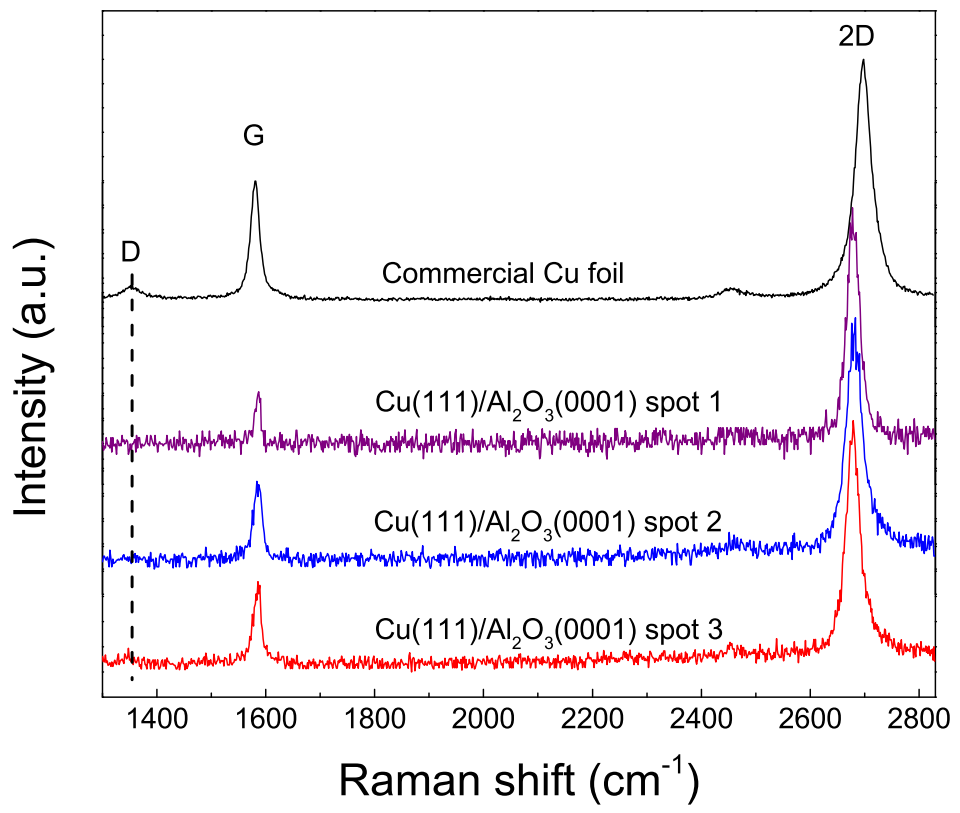

Figure 4: Raman spectra obtained from graphene grown on the $\mathrm{Cu}(111) / \mathrm{Al}_{2} \mathrm{O}_{3}(0001)$ prepared at the substrate temperature of $473 \mathrm{~K}$. Three scans were collected from different spots in the graphene film. A spectrum of the typical CVD graphene grown on a commercial copper foil was shown on the top for comparison.

of the graphene layer. On the other hand, the D band was clearly seen in the Raman spectrum of the CVD graphene grown on a commercial copper foil, as shown in the top plot of Figure 4. The SEM image in Figure 5b showed nonuniform contrast, revealing the variation in the thickness of the graphene sheet. The results indicate that the graphene grown on the epitaxial copper film has much higher quality compared with the ones that we have ever grown on the commercial copper foils.

\section{Conclusion}

We were able to prepare the smooth $\mathrm{Cu}(111)$ film on the $c$-plane of sapphire, with the surface RMS of $7.2 \mathrm{~nm}$, using thermal evaporation at a substrate temperature of $473 \mathrm{~K}$. The copper film was epitaxially grown along (111) orientation, with a two-domain structure in which the domains azimuthally rotated by $60^{\circ}$. The graphene film grown on this copper film was confirmed to have few defects that are undetectable by Raman spectroscopy, as evident by the absence of the $\mathrm{D}$ band around $1350 \mathrm{~cm}^{-1}$. Our work indicated that the epitaxial $\mathrm{Cu}(111)$ film on sapphire with a smooth surface produces high quality graphene. 

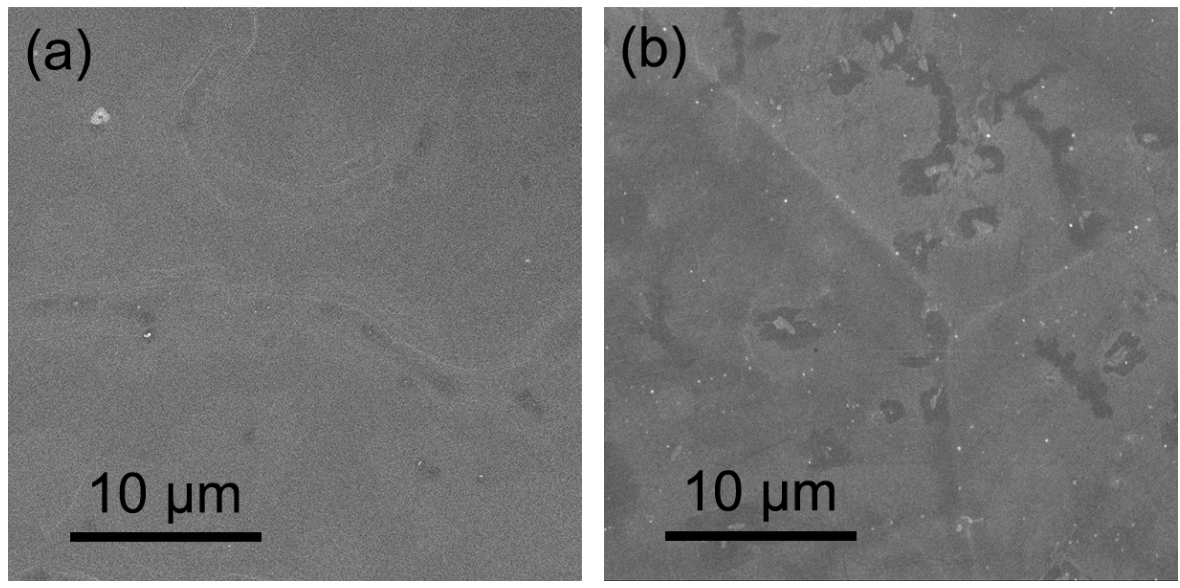

Figure 5: SEM images of the epitaxial $\mathrm{Cu}(111) / \mathrm{Al}_{2} \mathrm{O}_{3}(0001)$ film (a) and the commercial copper foil (b) after graphene growth. The dark contrast shows the thicker area of graphene.

\section{Acknowledgements}

The authors thank Prof. K. Gohara, Dr. K. Yamazaki, and Dr. Y. Maehara for their support on graphene growth. This work was partially supported by "Natural immunity" by the Ministry of Education, Culture, Sports, Science and Technology, Japan (MEXT) and "PEFC project" (P10001) by the New Energy and Industrial Technology Development Organization (NEDO).

\section{References}

[1] C. Lee, X. Wei, J. W. Kysar, J. Hone, Measurement of the elastic properties and intrinsic strength of monolayer graphene, Science 321 (5887) (2008) $385-388$.

[2] A. A. Balandin, S. Ghosh, W. Bao, I. Calizo, D. Teweldebrhan, F. Miao, C. N. Lau, Superior thermal conductivity of single-layer graphene, Nano Letters 8 (3) (2008) 902-907.

[3] S. Morozov, K. Novoselov, M. Katsnelson, F. Schedin, D. Elias, J. Jaszczak, A. Geim, Giant intrinsic carrier mobilities in graphene and its bilayer, Phys. Rev. Lett. 100 (1) (2008) 016602.

[4] T. Ma, K. Miyazaki, H. Ariga, S. Takakusagi, K. Asakura, Investigation of the cleanliness of transferred graphene: the first step toward its application as a window material for electron microscopy and spectroscopy, Bull. Chem. Soc. Jpn. 88 (8) (2015) 1029-1035.

[5] X. Li, W. Cai, J. An, S. Kim, J. Nah, D. Yang, R. Piner, A. Velamakanni, I. Jung, E. Tutuc, S. K. Banerjee, L. Colombo, R. S. Ruoff, Large-area 
synthesis of high-quality and uniform graphene films on copper foils, Science 324 (5932) (2009) 1312-1314.

[6] K. Kim, Z. Lee, W. Regan, C. Kisielowski, M. F. Crommie, A. Zettl, Grain boundary mapping in polycrystalline graphene, ACS Nano 5 (3) (2011) $2142-2146$.

[7] P. Y. Huang, C. S. Ruiz-Vargas, A. M. van der Zande, W. S. Whitney, M. P. Levendorf, J. W. Kevek, S. Garg, J. S. Alden, C. J. Hustedt, Y. Zhu, J. Park, P. L. McEuen, D. A. Muller, Grains and grain boundaries in singlelayer graphene atomic patchwork quilts, Nature 469 (7330) (2011) 389-92.

[8] H. Wang, G. Wang, P. Bao, S. Yang, W. Zhu, X. Xie, W.-J. Zhang, Controllable synthesis of submillimeter single-crystal monolayer graphene domains on copper foils by suppressing nucleation, J. Am. Chem. Soc. 134 (8) (2012) 3627-30.

[9] J. M. Wofford, S. Nie, K. F. McCarty, N. C. Bartelt, O. D. Dubon, Graphene islands on $\mathrm{Cu}$ foils: the interplay between shape, orientation, and defects, Nano lett. 10 (12) (2010) 4890-6.

[10] R. S. Edwards, K. S. Coleman, Graphene film growth on polycrystalline metals, Acc. Chem. Res. 46 (1) (2013) 23-30.

[11] L. Gao, W. Ren, H. Xu, L. Jin, Z. Wang, T. Ma, L.-P. Ma, Z. Zhang, Q. Fu, L.-M. Peng, X. Bao, H.-M. Cheng, Repeated growth and bubbling transfer of graphene with millimetre-size single-crystal grains using platinum, Nat. Commun. 3 (2012) 699.

[12] J. M. Wofford, E. Starodub, A. L. Walter, S. Nie, A. Bostwick, N. C. Bartelt, K. Thürmer, E. Rotenberg, K. F. McCarty, O. D. Dubon, Extraordinary epitaxial alignment of graphene islands on $\mathrm{Au}(111)$, New J. Phys. 14 (5) (2012) 053008.

[13] J.-H. Lee, E. K. Lee, W.-J. Joo, Y. Jang, B.-S. Kim, J. Y. Lim, S.-H. Choi, S. J. Ahn, J. R. Ahn, M.-H. Park, C.-W. Yang, B. L. Choi, S.-W. Hwang, D. Whang, Wafer-scale growth of single-crystal monolayer graphene on reusable hydrogen-terminated germanium., Science 344 (6181) (2014) 2869 .

[14] P. Procházka, J. Mach, D. Bischoff, Z. Lišková, P. Dvořák, M. Vaňatka, P. Simonet, A. Varlet, D. Hemzal, M. Petrenec, L. Kalina, M. Bartošík, K. Ensslin, P. Varga, J. Čechal, T. Šikola, Ultrasmooth metallic foils for growth of high quality graphene by chemical vapor deposition, Nanotechnology 25 (18) (2014) 185601.

[15] S. Dhingra, J.-F. Hsu, I. Vlassiouk, B. D'Urso, Chemical vapor deposition of graphene on large-domain ultra-flat copper, Carbon 69 (2014) 188-193. 
[16] K. M. Reddy, A. D. Gledhill, C.-H. Chen, J. M. Drexler, N. P. Padture, High quality, transferrable graphene grown on single crystal $\mathrm{Cu}(111)$ thin films on basal-plane sapphire, Appl. Phys. Lett. 98 (11) (2011) 113117.

[17] Y. Ogawa, B. Hu, C. M. Orofeo, M. Tsuji, K.-i. Ikeda, S. Mizuno, H. Hibino, $\mathrm{H}$. Ago, Domain structure and boundary in single-layer graphene grown on $\mathrm{Cu}(111)$ and $\mathrm{Cu}(100)$ films, J. Phys. Chem. Lett. 3 (2) (2012) 219-226.

[18] R. M. Jacobberger, M. S. Arnold, Graphene growth dynamics on epitaxial copper thin films, Chem. Mater. 25 (6) (2013) 871-877.

[19] S. Lee, J. Y. Kim, T.-W. Lee, W.-K. Kim, B.-S. Kim, J. H. Park, J.-S. Bae, Y. C. Cho, J. Kim, M.-W. Oh, et al., Fabrication of high-quality singlecrystal $\mathrm{Cu}$ thin films using radio-frequency sputtering, Sci. Rep. 4 (2014) 6230 .

[20] H. Bialas, E. Knoll, Heteroepitaxy of copper on sapphire under uhv conditions, Vacuum 45 (9) (1994) 959-966.

[21] M. Volmer, A. Weber, Novel growth mechanism in heteroepitaxial semiconductor growth, Z. phys. Chem 119 (1926) 277.

[22] D. Pashley, Epitaxy growth mechanisms, Mater. Sci. Technol. 15 (1) (1999) 2-8.

[23] L. Malard, M. Pimenta, G. Dresselhaus, M. Dresselhaus, Raman spectroscopy in graphene, Phys. Rep. 473 (5-6) (2009) 51-87. 